\title{
Extinction responding following partial reinforcement: The effects of number of rewarded trials and magnitude of reward
}

\author{
MICHAEL D. MORRIS and E. J. CAPALDI \\ Purdue University, Lafayette, Indiana 47907
}

\begin{abstract}
Rats were trained on a daily partial reward schedule of small magnitude of reward (S), nonreward $(\mathrm{N})$, and large magnitude of reward (L), which began with SN or SSNN for all animals. The remainder of the daily schedule was defined by the factorial combination of the number of rewards ( 1 vs. 3) and the magnitude of reward (S vs. L). Following 18 days of such training, 20 trials of extinction were administered. It was found that increasing the number of rewarded trials in a partial reinforcement schedule decreased resistance to extinction. Furthermore, increased number of large-magnitude rewards reduced resistance to extinction to a greater extent than increased number of small-magnitude rewards.
\end{abstract}

One major purpose of the present investigation was to determine the effect on resistance to extinction of the number of rewarded trials in a partial reinforcement schedule. The number of rewarded trials is of particular interest because several theories of instrumental learning and extinction suggest that expectancy of reward is an increasing function of the number of rewarded trials and that expectancy of reward influences resistance to extinction (Amsel, 1958; Capaldi, in press; Spence, 1960; Traupmann, Amsel, \& Wong, 1973; Wagner, 1963).

However, any attempt to relate partial reinforcement extinction to the number of rewarded trials faces a serious difficulty. Unfortunately, in all partial reinforcement investigations in which there was variation in the number of rewarded trials, confounding with other variables occurred. For example, in investigations concerned with the effects of percentage of reward on extinction (e.g., Bacon, 1962; Weinstock, $1954,1958)$, the number of rewarded trials was confounded with the number of nonrewarded trials and with such sequential variables as the number of times nonreward was followed by reward (N-R transition), the number of times reward was followed by nonreward ( $\mathrm{R}-\mathrm{N}$ transition), and the number of nonrewarded trials preceding reward ( $\mathrm{N}$-length-see Capaldi, 1966). In those investigations, as the number of rewards increased, the number of nonrewards and $\mathrm{N}$-length decreased while $\mathrm{N}-\mathrm{R}$ and $\mathrm{R}-\mathrm{N}$ transitions increased. In another set of investigations concerned with the effects on extinction of the level of

This research was supported in part by National Institute of Child Health and Development Grant SK HD 04379-03 to E. J. Capaldi. Requests for reprints should be sent to Michael D. Morris, Department of Psychology, Coe College, Cedar Rapids, lowa 52402 . partial reinforcement training, as the number of rewarded trials increased, so too did the number of nonrewarded trials and $\mathrm{N}-\mathrm{R}$ and $\mathrm{R}-\mathrm{N}$ transitions (e.g., McCain, Lee, \& Powell, 1962; Wagner, 1963).

Thus, there is little evidence to support the hypothesis that the number of rewarded trials in a partial reinforcement schedule influences resistance to extinction independently of other variables such as the number of nonrewards and a variety of sequential variables such as $\mathrm{N}$-length and N-R and $\mathrm{R}-\mathrm{N}$ transitions. Clearly, theories which suggest that the number of rewarded trials is an important determinant of resistance to extinction are founded upon tenuous empirical grounds. The present experiment was designed to remedy this situation by providing evidence as to the effect on extinction of the number of rewarded trials independently of the number of nonrewards and a variety of sequential variables.

A second major purpose of the present investigation was to examine the relationship between the number of rewarded trials and the magnitude of reward on extinction. Previous investigations on the effect of the level of training on extinction which also varied the magnitude of reward revealed greater reductions in resistance to extinction as a function of training level for large-magnitude-of-reward groups than for small-magnitude-of-reward groups (Campbell, Crumbaugh, Rhodus, \& Knouse, 1971; Capaldi \& Freese, 1974). It was of interest in the present investigation to discover whether magnitude of reward would produce similar effects when sequential variables were held constant. This was especially relevant since it had been previously suggested that increased number of large-reward $\mathrm{R}-\mathrm{N}$ transitions might reduce resistance to extinction (Capaldi, 1967).

To accomplish the purposes of this experiment, four groups of rats were trained on one of four 
schedules of partial reinforcement. Each daily schedule began with the same sequence of small magnitude of reward (S) and nonreward (N): SN or, on alternate days, SSNN. The remainder of the daily schedule was determined by the factorial combination of the number of rewards ( 1 vs. 3 ) with the magnitude of reward (S vs. L).

The predictions derived from the reinforcementlevel principle (Capaldi, 1974; Capaldi \& Freese, 1974) were that resistance to extinction would be a decreasing function of the number of rewarded trials and that this decrease would be greater for large magnitude of reward than for small magnitude of reward.

\section{METHOD}

\section{Subjects}

Forty male albino rats, 98 days old at the beginning of the experiment, were purchased from Holtzman Co., Madison, Wisconsin.

\section{Apparatus}

The apparatus was a runway, $208.4 \mathrm{~cm}$ long, $22.0 \mathrm{~cm}$ high, and $10.2 \mathrm{~cm}$ wide, with a hinged lid of $1.3-\mathrm{cm}$ hardware cloth and a wood floor. The alley was divided into three sections. The first section consisted of a treadle, $20.3 \mathrm{~cm}$ long, suspended above a microswitch. Placement of the front paws of the rat on the forward edge of the treadle started the first clock $(.01 \mathrm{sec})$. Interruption of a photobeam $5.1 \mathrm{~cm}$ beyond the treadle stopped Clock 1 (start time) and activated Clock 2 (run time). Interruption of a photobeam $132.1 \mathrm{~cm}$ beyond the first photobeam stopped Clock 2 and activated Clock 3 (goal time). The goal clock was stopped when a photobeam $39.4 \mathrm{~cm}$ beyond the second photobeam and $6.4 \mathrm{~cm}$ in front of a $5.1 \times 10.2 \times 3.8 \mathrm{~cm}$ food tray was broken. A $3.8 \times 3.2 \times 1.9 \mathrm{~cm}$ metal food cup was positioned on the right side of the food tray. A brass guillotine door, $30.5 \mathrm{~cm}$ from the end of the runway, was manually lowered to prevent retracing once an animal had interrupted the third photobeam.

\section{Procedure}

Upon arrival at the laboratory, rats were placed on an ad-lib feeding schedule for about 2 weeks. On Day 1 of the experiment, food was removed from the rats' cages. On the remaining days of the experiment, the rats were maintained on a 13-g daily food ration with water always available. On Day 9 and the 2 following days, the rats were handled in squads of four for $5 \mathrm{~min}$, after which they were returned to their home cages, where a glass caster filled with $20.045-\mathrm{g}$ Noyes pellets was provided for each rat. On the day following the last day of handling, the rats were randomly assigned to one of four treatment groups defined by the factorial combination of the magnitude of reward received following nonreward and the number of rewards following nonreward. Large reward magnitude (L) consisted of $20.045 \mathrm{~g}$ Noyes pellets, while small magnitude of reward (S) consisted of 1 pellet. Training schedules consisted of two different daily schedules for each group that were alternated on odd- and even-numbered days of acquisition. On odd-numbered days beginning with Day 1 of acquisition training, Group $1 \mathrm{~L}(\mathrm{n}=10)$ received an $S$ on Trial 1, N on Trial 2, and $\mathrm{L}$ on Trial 3 (represented as SNL); Group 1S $(n=10)$ received an SNS schedule; Group 3L $(n=10)$ received an SNLLL schedule; and Group $3 S(n=10)$ received an SNSSS schedule. On even-numbered days, the schedules for Groups 1L, 1S, 3L, and 3S were SSNNL, SSNNS, SSNNLLL, and SSNNSSS, respectively. On all rewarded trials, whether L or
$\mathrm{S}$, the rats were removed from the goalbox immediately after consuming all pellets. On $\mathrm{N}$ trials, the subjects were confined in the goalbox for $30 \mathrm{sec}$. Acquisition was continued for 18 days (72 trials for $1 \mathrm{~L}$ and $1 \mathrm{~S}$ groups and 108 trials for $3 \mathrm{~L}$ and $3 \mathrm{~S}$ groups).

Rats were run in squads of four, one from each treatment condition. At the beginning of each day, the rats within a squad were randomly assigned a running order. The first rat in the squad rotation did not receive its second trial until the other three rats had completed their first trial, and so on. This produced an intertrial interval of about 3 to $5 \mathrm{~min}$. On the last two trials of each day, only Groups $3 \mathrm{~L}$ and $3 \mathrm{~S}$ were run. As a result, the experimenter waited $1 \mathrm{~min}$ to allow for the time which would have been necessary to run rats of the $1 \mathrm{~L}$ or $1 \mathrm{~S}$ group.

After the last day of acquisition training, 2 days of massed extinction were administered at a rate of 10 trials per day. Squad rotation and confinement on $\mathbf{N}$ trials was the same as in acquisition training.

\section{RESULTS}

All three alley measures were added together to produce a total measure. Then all four measures were converted to speeds $(1 / \mathrm{sec})$. All analyses were performed upon these speeds.

The results of the experiment can be summarized almost completely by total runway speeds. Figure 1 presents the total speed for the four groups during acquisition in blocks of eight trials. These blocks represent the trials common to all groups during the day; that is, the first three trials on odd-numbered days and the first five trials on even-numbered days. It is apparent from this figure that responding was acquired more rapidly with increased number of rewards and increased reward magnitude. However, by the end of acquisition there were no differences between groups.

The total speeds were subjected to a factorial analysis of variance, with the number of rewards and the magnitude of reward as between-subject variables and trials (8) and blocks of trials (9) as repeated

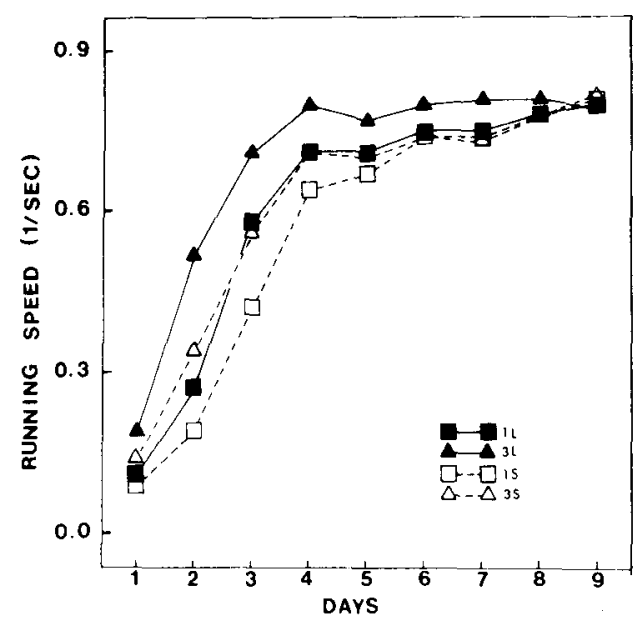

Figure 1. Mean total speeds in blocks of eight trials for the four groups on the common trials of acquisition. 


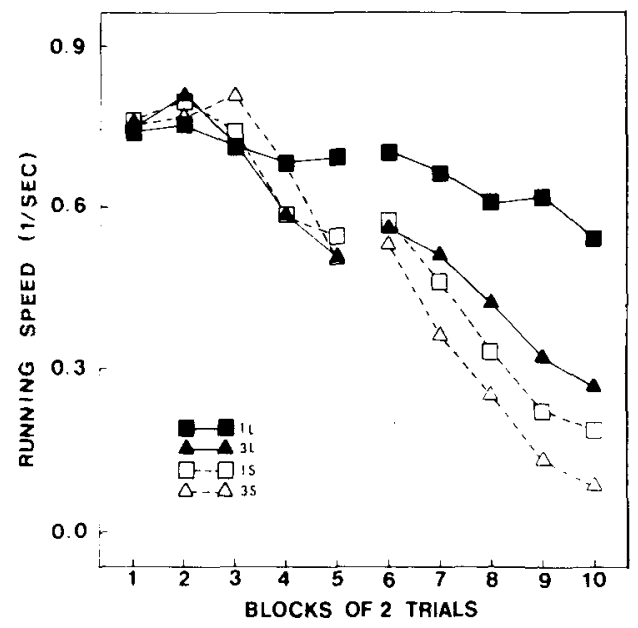

Figure 2. Mean total speeds in blocks of two trials for the four groups during the 2 days of extinction.

measures. The analysis indicated that there was a significant Number of Rewards by Blocks interaction $[\mathrm{F}(8 / 28)=5.12, \mathrm{p}<.001]$ and a significant Magnitude of Reward by Blocks interaction $[F(8 / 28)=$ $3.71, \mathrm{p}<.001]$. These interactions reflect the observation that while the groups differed early in acquisition, this difference disappeared on later blocks. Simple effects of the number of rewards and the magnitude of reward at the last block of eight trials revealed no significant differences $(\mathrm{Fs}<1)$.

Figure 2 depicts the extinction performance of the four groups. As can be seen, the groups did not differ much during the 1st day of extinction; however, during the 2 nd day, Groups $3 \mathrm{~L}$, 1S, and $3 \mathrm{~S}$ exhibited large decrements in responding while Group $1 \mathrm{~L}$ showed little evidence of extinction. From the standpoint of variables, this figure shows that increasing the number of rewarded trials decreased resistance to extinction and that this effect was greater for largemagnitude-of-reward groups (1L vs. 3L) than for small-magnitude-of-reward groups (1S vs. 3S).

Statistical analyses support these observations. A factorial analysis of variance with the number of rewards and the magnitude of reward as betweensubject variables and trials (10) and days (2) as repeated measures revealed that both the number of rewards $[F(1 / 36)=4.31, p<.05]$ and the magnitude of reward $[F(1 / 36)=7.46, p<.01]$ reached significance. While the Number of Rewards by Magnitude of Reward interaction was not reliable $[\mathrm{F}(1 / 36)=1.33$, $\mathrm{p}>.10$ ] for total speeds, a similar analysis of the goal-section speeds did find this interaction to be reliable $[F(1 / 36)=6.46, p<.01]$. Furthermore, a Newman-Keuls test of the four group means for total speeds indicated that while the difference between the two large-magnitude-of-reward groups (1L vs. 3L) was reliable $(p<.01)$, the difference between the two small-magnitude-of-reward groups was not.

\section{DISCUSSION}

In the present experiment, it was found that increasing the number of rewarded trials in a partial reinforcement schedule decreased resistance to extinction independently of the number of nonrewarded trials and sequential variables such as N-length. Furthermore, the effect of the number of rewards on extinction was somewhat greater when large magnitude of reward was employed than when small magnitude of reward was employed.

The present results have implications for evaluating the meaning of several classes of previous experimental findings. Thus, some investigations have found that increases in the percentage of reinforcement led to a decrease in resistance to extinction (Bacon, 1962; Weinstock, 1954, 1958). Since N-length covaried with the percentage of reinforcement in these investigations, it has been suggested that these results may be attributed to differences in $\mathrm{N}$-length (Capaldi, 1964). To support this view, experiments have been reported which demonstrated that decreases in $\mathrm{N}$-length produce decreases in resistance to extinction independently of the percent of reinforcement and the number of nonrewarded trials (Capaldi \& Kassover, 1970; Capaldi \& Stanley, 1965). The present results, however, suggest that $\mathrm{N}$-length is not the only variable relevant to results of studies manipulating the percentage of reinforcement. Since increases in the percentage of reward in these investigations led to an increase in the number of rewarded trials, studies wherein increases in the percentage of reinforcement lead to decrease in resistance to extinction could alternatively be explained on the basis of increased number of rewards. The present results may also explain why some investigators (Haggbloom \& Williams, 1971; Koteskey, 1969) found that increased percentage of reinforcement produced decreases in resistance to extinction even though sequential variables such as $\mathrm{N}$-length were controlled. In any event, it appears that variations in the percentage of reinforcement influence extinction responding through several variables, including $\mathrm{N}$-length and the number of rewarded trials.

The present results also have implications for evaluating the meaning of investigations that have examined the effect on extinction of varying the level of partial reward training. While the findings of those investigations have not proven to be totally consistent, increases in training level beyond 70 or so trials have led (generally) to a decrease in the resistance to extinction (Campbell et al., 1971; Capaldi \& Freese, 1974; McCain, Lee, \& Powell, 1962; Wagner, 1963). Furthermore, this decrease in resistance to extinction was greater for large magnitude of partial reward than for small magnitude of partial reward (Campbell et al., 1971; Capaldi \& 
Freese, 1974). The present results suggest that one reason for this overlearning extinction effect is that increased training increases the number of rewarded trials and thus results in a decrease in resistance to extinction. Moreover, the present findings suggest that if these additional rewarded trials are of a large magnitude, they would be expected to produce larger decreases in resistance to extinction. That the overlearning extinction effect is not consistently found with partial reward (e.g., Bacon, 1962; Hill \& Spear, 1962; Uhl \& Young, 1967) does not invalidate this reasoning, since other variables change as a result of increased training. Both the number of nonrewards and the number of $N-R$ transitions increase with increased training; increases in both of these variables are thought to increase resistance to extinction (Capaldi, 1966, in press). As a result, it may be that in investigations varying the training level, the effect of increased number of rewards on extinction is counteracted by the effect on extinction of increased number of nonrewards and N-R transitions. It might also be mentioned that some investigations (e.g., Hill \& Spear, 1962) employed small magnitude of reward, which would be expected, in light of the present results, to decrease the size of the overlearning extinction effect.

The finding that a block of continuous reinforcement, either preceding or following partial reinforcement, decreases resistance to extinction relative to partial reinforcement alone (Capaldi, 1974; Sutherland, Mackintosh, \& Wolf, 1965; Theios, 1962) also bears examination in relation to the present findings, since these procedures also involve an increase in the number of rewards. Previous theorizing has emphasized the sequence of continuous and partial reward in determining reduced resistance to extinction (see Sutherland \& Mackintosh, 1971); however, the present results suggest that the sequence of blocks of continuous and partial reinforcement may not be the only determinant of the extinction effects observed, since increased number of rewards alone is sufficient to produce this reduction in resistance to extinction. As a result, theories which rely on the sequence of blocks of partial reward and blocks of consistent reward as an explanation of these findings (e.g., Sutherland \& Mackintosh, 1971) must also consider the effects of increased number of rewards no matter when they occur during training.

An interpretation of the effects of increasing the number of rewards on resistance to extinction may be derived from the reinforcement level principle (Capaldi, 1974, in press). According to this view, animals develop an expectancy of reward which is an increasing function of the number of rewarded trials and the magnitude of reward and a decreasing function of the number of nonrewards. When this expectancy of reward exceeds the actual reward received on a trial, cues present on that trial gain inhibitory control of responding such that the greater the discrepancy between expectancy of reward and obtained reward, the greater the inhibitory growth. When the number of rewarded trials are increased in a partial reinforcement schedule, expectancy of reward would increase. This would lead to an increased discrepancy between expectancy of reward and nonreward and thus an increased growth of inhibition to cues present on nonrewarded trials during both acquisition and extinction. Since some of these cues (e.g., alley cues, memory stimulus of nonreward) also control extinction responding, increasing the number of rewarded trials would lead to a decrease in resistance to extinction. The reinforcement level principle also explains why this reduced resistance to extinction was greater with large magnitude of reward than with small. According to this theory, increasing the number of large-reward trials would increase expectancy of reward to a greater extent than increasing the number of small-reward trials. As a result, there would be a greater increase in inhibition for increased number of large-reward trials than for increased number of small-reward trials. Therefore, it would be expected that larger differences in extinction rate would be observed for large-magnitude groups than would be for smallmagnitude groups.

\section{REFERENCES}

Amsel, A. The role of frustrative nonreward in noncontinuous reward situations. Psychological Bulletin, 1958, 55, 102-119.

BAcon, W. E. Partial-reinforcement extinction effect following different amounts of training. Journal of Comparative and Physiological Psychology, 1962, 55, 998-1003.

Campbell, P. E., Crumbaugh, C. M. Rhodus, D. M., \& Knouse, S. B. Magnitude of partial reward and amount of training in the rat: An hypothesis of sequential effects. Journal of Comparative and Physiological Psychology, 1971, 75, 120-128.

CAPAldi, E. J. Effect of N-length, number of different $\mathrm{N}$-lengths, and number of reinforcements on resistance to extinction. Journal of Experimental Psychology, 1964, 68, 230-239.

Capaldi, E. J. Partial reinforcement: A hypothesis of sequential effects. Psychological Review, 1966, 73, 459-477.

CAPAldi, E. J. A sequential hypothesis of instrumental learning. In K. W. Spence \& J. T. Spence (Eds.), The psychology of learning and motivation (Vol, 1). New York: Academic Press, 1967.

CaPAldi, E. J. Partial reward either following or preceding consistent reward: A case of reinforcement level. Journal of Experimental Psychology, 1974, 102, 954-962.

Capaldi, E. J. Reinforcement level: An expectancy approach to relative reinforcement and nonreinforcement effects. In G. McCain \& J. Baerwaldt (Eds.), The Arlington Symposium on Learning. Stamford, Conn: Greylock, in press.

Capaldi, E. J., \& Freese, M. R. Partial reward training level and reward magnitude: Effects on acquisition and extinction. Learning and Motivation, 1974, 5, 299-310.

Capaldi, E. J., \& Kassover, K. Sequence, number of nonrewards, anticipation, and intertrial interval in extinction. Journal of Experimental Psychology, 1970, 84, 479-482. 
Capaldi, E. J., \& Stanley, L. R. Percentage of reward vs, N-length in the runway. Psychonomic Science, 1965, 3, 263-264.

Haggbloom, S. J., \& Williams, D. T. Increased resistance to extinction following partial reinforcement: A function of $\mathrm{N}$ length or percentage of reinforcement? Psychonomic Science, 1971, 24, 16-18.

Hill, W. F., \& Spear, N. E. Resistance to extinction as a joint function of reward magnitude and the spacing of extinction trials. Journal of Experimental Psychology, 1962, 64, 636-639.

KOTESKEY, R. L. The effect of unreinforced-reinforced sequences on resistance to extinction following partial reinforcement. Psychonomic Science, 1969, 14, 36.

McCain, G., Lee, P., \& Powell, N. Extinction as a function of partial reinforcement and overtraining. Journal of Compara. tive and Physiological Psychology, 1962, 55, 1004-1006.

Spence, K. W. Behavior theory and learning. Englewood Cliffs, New Jersey: Prentice-Hall, 1960.

Sutherland, N. S., Mackintosh, N. J., \& Wolf, J. B. Extinction as a function of the order of partial and consistent reinforcement. Journal of Experimental Psychology, 1965, 69, 56-59.

Sutherland, N. S., \& Mackintosh, N. J. Mechanisms of animal discrimination learning. New York: Academic Press, 1971.
The Los, J. The partial reinforcement effect sustained through blocks of continuous reinforcement. Journal of Experimental Psychology, 1962, 64, 1-6.

Traupman, K. L., Amsel, A., \& Wong, P. T. P. Persistance early and late in extinction as a function of number of continuous reinforcements preceding partial reinforcement training. Animal Learning \& Behavior, 1973, 1, 219-222.

Uht, C. N., \& Young, A. G. Resistance to extinction as a function of incentive, percentage of reinforcement, and number of nonreinforced trials. Journal of Experimental Psychology, 1967, 73, 556-564.

WAGner, A. R. Overtraining and frustration. Psychological Reports, 1963, 13, 717-718.

WEINSTOCK, S. Resistance to extinction of a running response following partial reinforcement under widely spaced trials. Comparative Journal of Physiological Psychology, 1954, 47, 318-322.

Weinstock, S. Acquisition and extinction of a partially reinforced running response at a 24 -hour intertrial interval. Journal of Experimental Psychology, 1958, 56, 151-158.

(Received for publication June 13, 1978; revision accepted March 18, 1979.) 